\section{Identification of melanoma initiating cells: does CD271 have a future?}

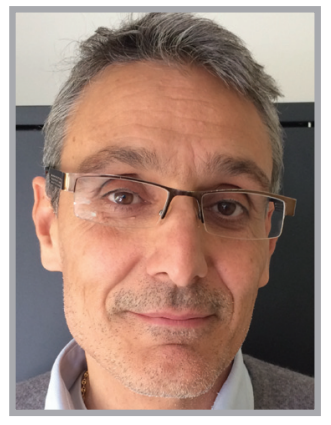

Robert Ballotti*
"Deciphering the role of CD271 in this plasticity

will allow better prognosis and may be the

treatment of melanoma, paving the road to a

further improvement of the melanoma

therapeutic armamentarium."

\section{Melanoma facts}

Melanoma is one of the few cancers whose incidence has constantly been increasing during the last decade. In fact, in Europe and in the USA more than 180,000 cases of melanoma will be diagnosed this year and more than 30,000 deaths from melanoma will occur. However, despite the efforts made in the prevention of the harmful effects of UV radiation of the solar light and the improvement in early diagnosis, no decrease in the mortality rate has been observed until the last couple of years.

This inability to improve the outcome of melanoma patient is due to the failure in therapeutic strategy until 2012. Indeed, since the approval of dacarbazine for metastatic melanoma treatment, by the US FDA in 1975 no further improvement in term of patient survival has been achieved, until 2011. Then, a breakthrough in melanoma treatment was brought by the use of antagonist antibodies targeting the negative immune check points (CTLA4, then PD1) that allowed a marked increase in the response rate (15-35\%), including complete and long lasting responses [1]. By the end of 2011, the first in class BRAF inhibitor induced a shift in the paradigm of melanoma treatment. Before this tarto kill cancer cells and shrink the tumors. In patients with metastatic melanoma harboring a $B R A F^{\mathrm{V} 600}$ mutation $(50 \%$ of the melanomas) the BRAF inhibitors effectively kill cancer cells resulting in about $80 \%$ of objective response with a spectacular decrease in the tumor burden [1]. However, the euphoria caused by the first result obtained with BRAF inhibitors was rapidly weakened by the appearance of frequent resistance and of disease relapse [1]

The assessment of the results obtained with these last generation drugs in melanoma treatment provided a rather favorable balance. However, the existence of primary and acquired resistance to these treatments has prompted the search for novel therapeutic strategies and new prognosis markers for drug responses.

\section{Why melanoma cells are or have become resistant}

Among several hypotheses that might explain the resistance of melanoma to current therapies, the trendiest one proposes the existence of a distinct melanoma cell population endowed with high geted therapy, the drugs were inefficient

*INSERM U1065, Equipe 1, Biology \& Pathologies of Mélanocytes, Equipe labellisée Ligue 2013, Centre Méditerranéen de Médecine Moléculaire, Université de Nice Sophia-Antipolis, UFR Médecine, Nice, France; ballotti@unice.fr

\section{KEYWORDS}

- drug resistance • melanoma

- plasticity • surface marker

- tumorigenicity
"Melanoma is one of the few cancers whose incidence has constantly been increasing during the last decade."

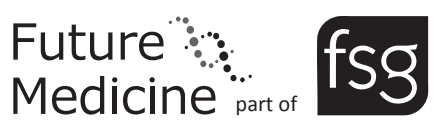


"Within the same tumor or the melanoma cell line, there are populations expressing different levels of CD271, endowed with different biological properties, while the phenotypic plasticity of melanoma cells enables a reversible transition between the two populations." tumorigenic properties. This population would account for the well-recognized melanoma heterogeneity, invasiveness and drug resistance. The functional capacities that melanoma cells should acquire to allow tumor development are probably associated with specific gene-expression profile that sustains these specific biological functions. Therefore, the characterization of these markers identifying the highly tumorigenic melanoma cells (called melanoma initiating cells [MICs]) is of paramount importance to improve current therapeutic strategies. However, to date, no consensual marker for MICs has been identified. High levels of CD20 [2], CD24 [3], ALDH [4], CD271 [5], ABCB5 [6] or JARID1B [7] have been reported in this population. The MICs have also been characterized by a low MITF expression [8] and a transient slow growing rate $[7,8]$.

\section{CD271 as a marker for melanoma aggressiveness}

Among these markers, CD271 has been subjected to the most intensive studies, since CD271 is a marker of neural crest cells from which melanocytes are derived. Thus, CD271 can also be considered as a melanoma dedifferentiation marker, which is generally correlated with aggressiveness. CD271, also known as NGFR, is a low-affinity receptor for NGF, a member of the neurotrophin family proteins. An initial wave of studies on CD271 in melanoma had already brought solid pieces of evidence on the involvement of CD271 in melanoma biology. In 1984, Ross et al. characterized this receptor as a $75 \mathrm{kDa}$ surface protein that is responsible for the lowaffinity binding of neurotrophins and NGF [9]. CD271 appears to be the only NGF receptor on melanomas since NTRK1 (TRKA) the high-affinity NGF receptor is absent from basically all melanoma cell lines [10], even thought, Truzzi et al. reported the expression of TRK1 on six melanoma cell lines [11]. The heterogeneity of CD271 expression on melanoma cells was first illustrated by studying its expression in 34 different cell suspensions from human melanoma metastasis [12]. Further cells expressing high level of CD271 displayed increased survival properties compared with cell expressing low level of CD271, sorted from the same melanoma cell line [13].

The most striking demonstration of the key role of CD271, in the identification of highly tumorigenic melanoma cells, was brought by Boiko et al. In this report, they clearly demonstrated that the CD271-positive melanoma cells had a high tumorigenic potential compared with CD217-negative cells originating from the same tumor, when injected into immuno-deficient mice [5]. These data were not confirmed by the report of Quintana et al. showing that $\mathrm{CD} 271^{+}$or $\mathrm{CD} 271^{-}$cells isolated from human melanoma metastases had the same tumorigenic potential [14]. Since then, compelling evidence have been gathered by different groups demonstrating the exacerbated tumorigenic potential of CD271 $1^{+}$melanoma cells [15-17] that might be explained by an increased expression of stemness and mesenchymal markers [16].

Furthermore, CD271 has been also validated as a marker for circulating melanoma cells that are thought to be endowed with high dissemination capacity [18]. Circulating melanoma cells seem to be a good prognosis marker for survival and BRAFi response. CD271-expressing cells may have an immune privilege, since CD271 covaries with PDL1. CD271 appears to be usually regulated by different stress conditions, such as stringent culture conditions, hypoxic conditions, low glucose concentration or drug (BRAFi or dacarbazin) exposure [17]. Epigenetic modifications seem to play a key role in the increase in the $\mathrm{CD} 271^{+}$population [19]. This epigenetic regulation may allow some persistence of the phenotype, even after the cessation of the environmental inducer signal, but it may also allow a reversion to the initial phenotype through the so-called phenomenon of phenotypic switch or plasticity

However, these data do not mean that melanoma have a fixed hierarchical organization. The $\mathrm{CD} 271^{+}$cells that have an increase tumorigenic potential are able to undergo a phenotypic reversion to the CD271 phenotype and vice versa [16]. Since $\mathrm{CD} 271^{+}$population may vary during the tumor history, the phenotypic plasticity may be a major hurdle for the use of CD271 as a reliable prognosis marker. However, using a cohort of 30 patients, Beretti et al. showed that CD271 expression is inversely correlated with the patients survival [20]. To my knowledge, no correlation could be observed in DNA microarray analysis of larger series.

\section{CD271 as a possible therapeutic target}

Whether CD271 is only a marker of melanoma dedifferentiated or CD271 plays an active role in the aggressive phenotype of CD271+ melanoma cells remain to be clearly elucidated. CD271 
belongs to the TNF- $\alpha$ receptor family and is associated with a complex signalization network. Besides its specific neuronal functions, CD271 has been also involved in general cellular processes such as cell cycle, survival and migration. However, the existence of various membrane coreceptors, such as TRK or sortilin, as well as different signaling modules, allows CD271 to mediate divergent cellular effects such as survival and apoptosis. The nature of the coreceptors and of the signaling platform has been documented in PC12 or Schwann cells, but not in melanoma cells. Of note, NGF was shown to activate the SRC family member, YES, probably through NGFR [21]. In melanoma, the YES kinase was recently shown to control the expansion of the $\mathrm{CD} 24^{+}$population that showed an increased tumorigenic behavior compared with its CD24counterpart [3]. In melanoma cells, NGFR has been involved in migration and invasion induced by neurotrophins. A special attention has been brought to brain metastases and perineural invasions [22]. From these reports, it should be concluded that neurotrophin production by neural cells may favor melanoma cells invasion and development. CD271 is a validated marker for spindle cell melanoma and desmoplastic melanomas, the latter having a recognized neurotropism propensity [23]

Few studies have reported a role of NGFR in growth or survival of melanoma cells. A clear demonstration of the functional role of NGFR in tumor growth was brought by Redmer et al., showing that NGFR silencing prevents melanoma tumor development in xenograft model [24].

\section{What future for CD271 in melanoma prognosis \& treatment?}

As a whole, all the observations reported above constitute a convergent bundle of clues which places CD271 at the center of melanoma metastasis, resistance to treatment and even escape to the immune system. Therefore, CD271 might be a valuable prognosis marker for metastasis

\section{References}

1 Eggermont AM, Spatz A, Robert C. Cutaneous melanoma. Lancet 383(9919), 816-827 (2014).

2 Fang D, Nguyen TK, Leishear K et al. A tumorigenic subpopulation with stem cell properties in melanomas. Cancer Res. 65(20), 9328-9337 (2005).

development but also for response to targeted and immune therapies. The final validation of the role of CD271 as a marker of melanoma in aggressiveness must wait the CD271 expression analysis in larger series of patients with high-quality clinical data.

Beyond its use as a prognostic marker, CD217 is also a signaling platform, whose commitment can alter the biology of melanoma. As such, CD271 could be a direct therapeutic target of agonist or antagonist antibodies. The validation of this hypothesis requires further evaluation of the role of CD271 in melanoma development, using targeted and inducible CD271 knockout mouse model or melanoma cell line engineered to overexpress or downregulate CD271 expression

\section{Conclusion}

Within the same tumor or the melanoma cell line, there are populations expressing different levels of CD271, endowed with different biological properties, while the phenotypic plasticity of melanoma cells enables a reversible transition between the two populations. These observations have drawn the outlines of a new paradigm for melanoma development that should take into account modifications of melanoma cell properties during the history of tumor development. Deciphering the role of CD271 in this plasticity will allow better prognosis and may be the treatment of melanoma, paving the road to a further improvement of the melanoma therapeutic armamentarium.

\section{Financial \& competing interest disclosure}

The author has no relevant affiliations or financial involvement with any organization or entity with a financial interest in or financial conflict with the subject matter or materials discussed in the manuscript. This includes employment, consultancies, honoraria, stock ownership or options, expert testimony, grants or patents received or pending, or royalties.

No writing assistance was utilized in the production of this manuscript.

3 Liu W, Monahan KB, Pfefferle AD et al. LKB1/STK11 inactivation leads to expansion of a prometastatic tumor subpopulation in melanoma. Cancer Cell 21(6), 751-764 (2012).

4 Boonyaratanakornkit JB, Yue L, Strachan LR et al. Selection of tumorigenic melanoma cells using ALDH. J. Invest. Dermatol. 130 (12), 2799-2808 (2010).
5 Boiko AD, Razorenova OV, van de Rijn M et al. Human melanoma-initiating cells express neural crest nerve growth factor receptor CD271. Nature 466(7302), 133-137 (2010).

6 Schatton T, Murphy GF, Frank NY et al. Identification of cells initiating human melanomas. Nature 451(7176), 345-349 (2008). 
7 Roesch A, Fukunaga-Kalabis M, Schmidt EC et al. A temporarily distinct subpopulation of slow-cycling melanoma cells is required for continuous tumor growth. Cell 141(4), 583-594 (2010).

8 Cheli Y, Giuliano S, Botton T et al. Mitf is the key molecular switch between mouse or human melanoma initiating cells and their differentiated progeny. Oncogene 30 (20), 2307-2318 (2011).

9 Ross AH, Grob P, Bothwell M et al. Characterization of nerve growth factor receptor in neural crest tumors using monoclonal antibodies. Proc. Natl Acad. Sci. USA 81(21), 6681-6685 (1984).

10 Cancer Cell Line Encyclopedia. www.broadinstitute.org

11 Truzzi F, Marconi A, Lotti R et al. Neurotrophins and their receptors stimulate melanoma cell proliferation and migration. J. Invest. Dermatol. 128(8), 2031-2040 (2008).

12 Berd D, Herlyn M, Koprowski H, Mastrangelo MJ. Flow cytometric determination of the frequency and heterogeneity of expression of human melanoma-associated antigens. Cancer Res. 49(23), 6840-6844 (1989).

13 Marchetti D, Aucoin R, Blust J, Murry B, Greiter-Wilke A. p75 neurotrophin receptor functions as a survival receptor in brainmetastatic melanoma cells. J. Cell. Biochem. 91(1), 206-215 (2004).

14 Quintana E, Shackleton M, Sabel MS et al. Efficient tumour formation by single human melanoma cells. Nature 456(7222), 593-598 (2008).

15 Civenni G, Walter A, Kobert N et al. Human CD271-positive melanoma stem cells associated with metastasis establish tumor heterogeneity and long-term growth. Cancer Res. 71(8), 3098-3109 (2011).

16 Cheli Y, Bonnazi VF, Jacquel A et al. CD271 is an imperfect marker for melanoma initiating cells. Oncotarget 5(14), 5272-5283 (2014).

17 Ravindran Menon D, Das S, Krepler C et al. A stress-induced early innate response causes multidrug tolerance in melanoma. Oncogene doi:10.1038/onc.2014.372 (2014) (Epub ahead of print).

18 Klinac D, Gray ES, Freeman JB et al. Monitoring changes in circulating tumour cells as a prognostic indicator of overall survival and treatment response in patients with metastatic melanoma. BMC Cancer 14, 423 (2014).

19 Li S, Yue D, Chen X et al. Epigenetic regulation of CD271, a potential cancer stem cell marker associated with chemoresistance and metastatic capacity. Oncol. Rep. 33(1), 425-432 (2015).

20 Beretti F, Manni P, Longo C et al. CD271 is expressed in melanoma with more aggressive behavior with correlation of characteristic morphology in in vivo reflectance confocal microscopy. Br. J. Dermatol. 172(3), 662-668 (2015).

21 Marchetti D, Parikh N, Sudol M, Gallick GE. Stimulation of the protein tyrosine kinase c-Yes but not c-Src by neurotrophins in human brain-metastatic melanoma cells. Oncogene 16(25), 3253-3260 (1998).

22 Marchetti D, Nicolson GL. Neurotrophin stimulation of human melanoma cell invasion: selected enhancement of heparanase activity and heparanase degradation of specific heparan sulfate subpopulations. $A d v$. Enzyme Regul. 37, 111-134 (1997).

23 Ordonez NG. Value of melanocytic-associated immunohistochemical markers in the diagnosis of malignant melanoma: a review and update. Hum. Pathol. 45(2), 191-205 (2014).

24 Redmer T, Welte Y, Behrens D et al. The nerve growth factor receptor CD271 is crucial to maintain tumorigenicity and stem-like properties of melanoma cells. PLoS ONE 9(5), e92596 (2014). 